\title{
Autoeficacia, enfoque y papel de la práctica de los maestros en la enseñanza de la escritura
}

\author{
Deilis I. Pacheco, Jesús-Nicasio García y Carmen Díez \\ Universidad de León (España)
}

\begin{abstract}
Se presenta un estudio sobre el análisis de la práctica del profesor en la enseñanza de la escritura. La muestra estuvo compuesta por 137 profesores del área de lengua pertenecientes a 30 centros educativos de tercer ciclo de Educación Infantil, y de los tres ciclos de Educación Primaria. Se aplicó un instrumento (PRAES) que incluía la evaluación de la concepción teórica que tienen de cómo aprenden los alumnos, las creencias sobre los efectos de estas instrucciones, la corrección de la escritura y los métodos de enseñanza (formal y natural), así como la auto-eficacia del profesor en relación con dicha enseñanza. Los datos indican que el PRAES presenta una fiabilidad y validez adecuadas, además de proporcionar una descripción precisa del problema, indicándose que los profesores manifiestan valoraciones positivas en las escalas, pudiéndose predecir ciertas variables relevantes a partir del instrumento. Por último, se discuten y valoran los resultados y sus implicaciones, además de explicitar las limitaciones, indicándose, como deseable, realizar estudios que minimicen estos problemas, además de hacerlo no sólo en escritura, sino también en otras áreas.
\end{abstract}

Palabras clave: Autoeficacia, enfoque teórico, enseñanza escritura, práctica del profesor.

Self-efficacy, approach, and teacher's practice in the writing teaching. We present a research about the analysis of the teacher's practice in the teaching of the writing. The sample was composed by 137 teachers from language area, working in 30 different Kindergarten's schools and Primary Schools. We applied a device (PRAES) which include the theoretical approach that teachers have about how the students learn, the believes about the effects of those instructions, the writing correction, the teaching methods (formal and natural), and the teacher's self-efficacy related with those teaching. Data suggest that the PRAES presents an adequate reliability and validity, besides it provides a precise description of the problem; signaling that the teachers show positive opinion in the device, and we can predict some variables from the instrument. And last, we discuss and comments results and its implications. We discuss the shortcomings; and we signal as desirable to implement researches which reduce these problems; besides, we suggest to extend this type of study in other fields, not only in writing.

Key words: Self-efficacy, theoretical approach, teaching in writing, teacher's practice.

Correspondencia: Jesús-Nicasio García. Departamento de Psicología, Sociología y Filosofía. Área Psicología Evolutiva y de la Educación. Universidad de León. Campus de Vegazana s/n, C.P. 24071. León (España). E-mail: jn.garcia@unileon.es 
En cuanto a la escritura propiamente dicha, la revisión de estudios empíricos internacionales ha evidenciado el uso de técnicas, estrategias e instrumentos para animar, tanto a los profesores en ejercicio de la profesión, como a los futuros profesores, a reflexionar sobre su práctica profesional. Cuando se explora el papel y la importancia de diferentes estrategias en el desarrollo de las habilidades reflexivas de los profesores, a través de instrumentos como el cuestionario y la entrevista, se examina, al mismo tiempo, la influencia de sus enfoques, su pensamiento, sus posiciones y comentarios sobre la escritura (Bain, Mills, Ballantyne y Packer, 2002).

En este sentido, se han elaborado, validado e implementado, instrumentos que miden el papel de la práctica del profesor en el aula, y han permitido extraer datos relevantes sobre el tema, tales como la escala de orientación de la escritura -Writing Orientation Scale- de Graham, Harris, McArthur y Fink (2002) que analiza y evalúa las creencias de los profesores sobre los métodos de enseñanza formal e informal de la escritura; o la escala de eficacia del profesor para la escritura -Teacher Efficacy Scale for Writing- de Graham, Harris, Fink y MacArthur (2001) que describe la autoeficacia personal y general en la enseñanza, o la escala de prácticas de escritura del profesor The Teacher Writing Practices Scale-, de Graham et al. (2002) que permite obtener información sobre las habilidades de la escritura y los procesos de instrucción utilizados por los profesores en sus clases. Estos cuestionarios son útiles para evaluar, tanto la orientación teórica de los profesores con relación al proceso de enseñanzaaprendizaje de la escritura, como también los procedimientos y actividades que generalmente se siguen en la enseñanza de la escritura, es decir, el tipo de prácticas que se desarrollan en clase desde tres dimensiones diferentes, (i) la forma de pensar sobre los efectos de estas instrucciones, (ii) la corrección de la escritura y (iii) los métodos naturales de aprendizaje.

Los datos obtenidos en la revisión de estudios, evidenciaron que las creencias de los profesores ejercen una fuerte influencia en las prácticas y en el éxito de los alumnos en el aula (Graham et al., 2002). En el área de lengua, por ejemplo, algunas teorías indican que las prácticas de los profesores en el aula son modeladas por sus concepciones teóricas o creencias que ellos tienen sobre la instrucción en lectoescritura. Por consiguiente, el conocimiento de las orientaciones y enfoques teóricos sobre la instrucción de los profesores es un elemento importante para entender los procesos de enseñanza y aprendizaje (Graham et al., 2002, 2001).

Al relacionar la eficacia y las creencias de los profesores en la enseñanza de la escritura, los resultados son compatibles con la afirmación de que aquellos profesores con más sentido humanista y un menor enfoque de control, tienen un alto sentido de eficacia en la enseñanza. Los autores piensan que la eficacia de los profesores es un elemento importante para una enseñanza efectiva en el área de la escritura (Graham et al., 2002, 2001; Troia y Maddox, 2004, 2006). 


\section{Objetivo e hipótesis}

El objetivo de esta investigación se centra en estudiar y evaluar, por una parte, los procesos y componentes de la escritura desde el punto de vista que expresa el profesor (la motivación del alumno, el papel que juegan los padres o entorno familiar en este proceso, el uso de estrategias, procedimientos e instrumentos que utiliza el profesor en la enseñanza de la escritura); y por la otra, la orientación teórica de los profesores (creencias, atribuciones y expectativas, sentido de eficacia personal) en torno al proceso de enseñanza-aprendizaje de la escritura.

Estudiar los componentes que influyen en la práctica y las orientaciones teóricas que tienen los profesores (sus creencias, atribuciones y expectativas, sentido de eficacia personal) en torno al proceso de enseñanza-aprendizaje de la composición escrita, permitieron formular como primera hipótesis que el tipo de prácticas que los profesores desarrollan en clase son determinadas y obedecen a factores relacionados con la concepción que tienen de cómo aprenden los alumnos, la forma de pensar sobre los efectos de estas instrucciones, la corrección de la escritura y los métodos de enseñanza (formal y natural) utilizados para el aprendizaje de la escritura. En este sentido, uno esperaría que las opiniones, el enfoque teórico, lo que dicen que hacen los profesores, así como sus creencias de auto-eficacia se maticen en función del nivel educativo de los alumnos, y en función de la tipología de los mismos.

\section{METODO}

\section{Participantes}

En este estudio participaron 137 profesores y/o maestros, pertenecientes a 30 centros educativos públicos y privados de la provincia de León (ver tabla 1), que imparten clases en el área de lengua y tienen a su cargo a los alumnos con y sin dificultades de aprendizaje y/o bajo rendimiento del último ciclo de Educación Infantil y de los tres ciclos de Educación Primaria. El criterio de selección de esta muestra obedece a estudios previos, relativos al papel de la memoria operativa y de la atención en alumnos de diferentes edades y adecuadamente escolarizados, con y sin Déficit de Atención (DA) y con y sin Trastorno por Déficit de Atención con Hiperactividad (TDAH), que el Equipo de Investigación dirigido por el IP J.N. García en la Universidad de León, viene desarrollando en estos centros en torno a diferentes focos de preocupación relacionados con la composición escrita (ver García y Marbán, 2002; 2003; García et al., 2006; García y Arias-Gundín, 2004, 2005a, 2005b; García y de Caso, 2002, 2004, 2006a, 2006b; García y Fidalgo, 2003a; 2003b, 2004, 2006a, 2006b; García y González, 2006; García, Marbán y de Caso, 2001; García y Martínez, 2005; García y Rodríguez, 2007). 
De estos 137 profesores el 63,5\% eran mujeres, el 64,23\% trabajan en escuelas públicas y $37,77 \%$ en escuelas privadas. La edad media de los profesores es de 46,92 años, con un máximo de 67 y un mínimo de 23 años, indicando madurez y años de estabilidad en la docencia. El promedio de experiencia de los docentes en el campo laboral era de 23,43 años, lo que indica una gran experiencia docente, siendo ésta en el área de Lengua de 18,84 años, lo cual hace suponer que son muy conocedores de los aspectos instruccionales relacionados, probablemente, con la enseñanza de la composición escrita. Sólo 17 profesores $(12,4 \%)$ tenían el grado de licenciado en filología o pedagogía, los profesores restantes tenían el grado de maestro.

Tabla 1. Distribución de la muestra de profesores por género y ciclo educativo

\begin{tabular}{lcccc}
\hline \multicolumn{1}{c}{ Nivel educativo } & \multicolumn{2}{c}{ Género } & No responden & \multirow{2}{*}{ Total de profesores } \\
& MUJER & HOMBRE & estos datos & $8(5,83 \%)$ \\
\hline Tercer ciclo de Infantil & 7 & 0 & 1 & $35(25,54 \%)$ \\
Primer ciclo de Primaria & 24 & 6 & 5 & $44(32,11 \%)$ \\
Segundo ciclo de Primaria & 27 & 9 & 8 & $50(36,49 \%)$ \\
Tercer ciclo de Primaria & 29 & 10 & 11 & 137 \\
\hline TOTAL DE PROFESORES & 87 & 25 & 25 & $(18,24 \%)$ \\
& $(63,5 \%)$ & $(18,24 \%)$ & & \\
\hline
\end{tabular}

\section{Instrumentos}

La elaboración del instrumento que se presenta, se inició, por una parte, con la revisión de las bases de datos en Academic Search Elite, bases disponibles en la Biblioteca Central de la Universidad de León. Por otra parte, se hizo imprescindible la lectura y revisión de los Reales Decretos, Programas y Guías educativas donde se establecen, tanto el currículo de la Educación Infantil, como de la Educación Primaria, a fin de obtener información sobre los criterios de evaluación del área de Lengua en cada uno de los ciclos estudiados y, en concreto, de aquellos datos de estudios empíricos que se encuentran relacionados con el foco de estudio de la presente investigación.

Estas bases teóricas permitieron proseguir con la recopilación, traducción y, en su caso, adaptación de instrumentos, así como la elaboración de nuevos instrumentos que evaluaran los ámbitos y componentes que inciden en la práctica del profesor en la composición escrita. Una vez construidos, fueron sometidos a una validación (fiabilidad, validez, normas, etc.), por parte del equipo de investigación.

De esta manera, se obtuvo el instrumento que evalúa el Papel de la Práctica de los Profesores en la Escritura: PRAES, con sus respectivos cuestionarios: el primero, se centra en la opinión (PRAESPO); el segundo, en el enfoque teórico (PRAESPE); el tercero, se refiere a la actividad en el aula o a lo que efectivamente hacen (PRAESPA); y, por último, en la autoeficacia (PRAESAE). 


\section{Descripción general del significado psicológico que evalúan}

El PRAES, está conformado, tanto por los datos de Identificación del profesor, como por cuatro partes o cuestionarios relacionadas con los componentes y factores que afectan a la práctica: PRAESPO, PRAESPE, PRAESPA y PRAESAE. Para más detalles, ver tabla 2.

La nomenclatura utilizada para referirse e identificar cada cuestionario, según el orden secuencial de presentación en el protocolo del PRAES, es la siguiente:

Práctica-Opinión (PRAESPO). Hace referencia a las opiniones del profesor respecto al papel de la práctica en la escritura, considerando los siguientes componentes e indicadores: el componente personal del alumno (motivación, planificación y revisión); el componente de la práctica; el componente de la familia; y, el componente de la formación del profesorado. Este cuestionario fue elaborado específicamente por el equipo de investigación.

Práctica-Enfoque (PRAESPE). Evalúa la orientación teórica de los profesores en relación con la enseñanza formal y con el aprendizaje natural en el proceso de enseñanza-aprendizaje de la escritura. Este instrumento fue adaptado a partir de la escala The Writing Orientation Scale de Graham et al. (2001).

Práctica-Aula (PRAESPA). Mide las habilidades, los procedimientos instruccionales, las actividades, los materiales utilizados habitualmente por los profesores en la enseñanza de la escritura y, por último, la tipología textual o los textos que utilizan. Este instrumento, en parte, fue construido y elaborado por el equipo y, en parte, adaptado y traducido de Graham et al. (2001), a partir de su instrumento The Teacher Writing Practices Scale.

Práctica-Docencia (PRAESAE). Ayuda a comprender mejor el tipo de factores que crean dificultades personales y generales a los profesores en su actividad docente, determinando así sus creencias de autoeficacia en el proceso de enseñanza de la escritura. El instrumento diferencia dos tipos de autoeficacia, una personal y otra general. Este instrumento, fue desarrollado por el equipo de investigación, a partir de la Teacher Efficacy Scale for Writing de Graham et al. (2001), adaptado según las directrices y guías para la construcción de escalas de autoeficacia de Bandura (2005).

\section{Procedimiento}

La investigación que se presenta, enmarcada dentro de los estudios descriptivos, se inició, por una parte, con la revisión de las bases de datos en Academic Search Elite y, en concreto, con aquellos estudios empíricos que se encuentran relacionados con el foco del estudio, bases disponibles en la Biblioteca Central de la Universidad de León. Por otra parte, se hizo imprescindible la lectura y revisión de los Reales Decretos, Programas y Guías educativas donde se establecen tanto el currículo de la Educación Infantil como de la Educación Primaria, a fin de obtener información 
sobre los criterios de evaluación del área de lengua en cada uno de los ciclos estudiados.

Taba 2. Descripción de las pruebas para el estudio del Papel de la Práctica de los profesores

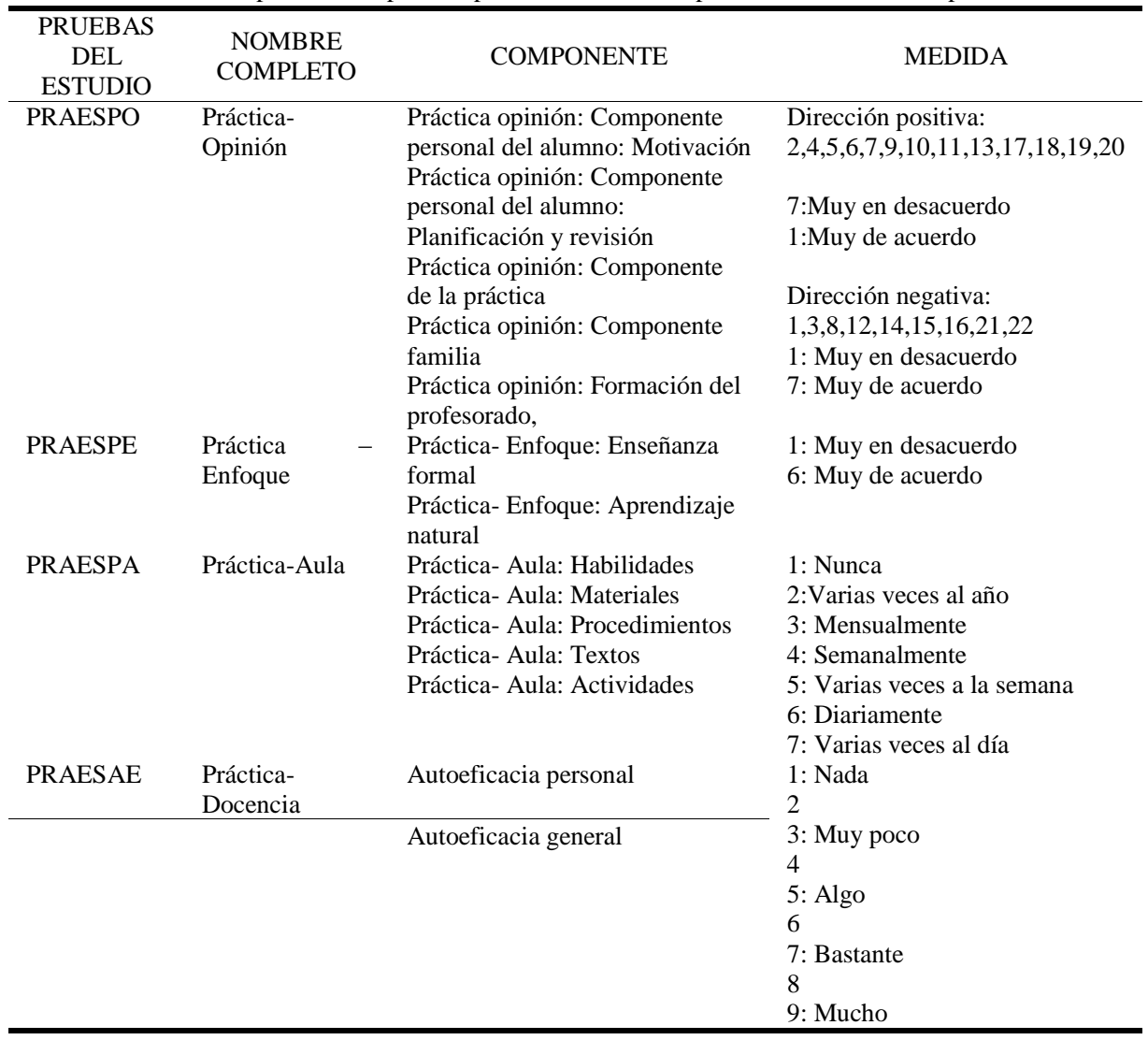

Estas bases teóricas permitieron proseguir con la recopilación, traducción y, en su caso, adaptación de instrumentos, así como la elaboración de nuevos instrumentos que evaluaran los ámbitos y componentes que inciden en la práctica de la composición escrita. Una vez construidos, fueron sometidos a una validación (fiabilidad, validez, normas, etc), por parte del Equipo de Investigación dirigido por el IP J.N. García. De esta manera, se obtuvo el protocolo: El Papel de la Práctica en la Escritura (PRAES), con sus respectivos cuestionarios.

A continuación, el diseño y plan de muestreo consistió en verificar el tiempo de aplicación de este protocolo en los centros educativos de la provincia de León, como aplicación piloto para eliminar los problemas y dificultades que originara y ajustarlo a 
las necesidades de la investigación. La muestra seleccionada, a partir del criterio explicitado anteriormente en el apartado de los participantes, fueron los profesores que tenían a su cargo alumnos con y sin dificultades de aprendizaje y/o bajo rendimiento de Educación Infantil y Primaria.

El siguiente paso fue el trabajo de campo, propiamente dicho. El mismo consistió, primeramente, en establecer contacto telefónico con los directores de los centros educativos para la debida autorización de visitar y aplicar el protocolo a los profesores del área de lengua, de acuerdo a los cursos seleccionados en cada uno de estos centros. Luego, dos investigadores del equipo (D.I. Pacheco y C. Díez), se desplazaron a los centros en las fechas y horas concertadas para que los profesores rellenaran los protocolos. La recogida de la muestra se hizo directamente por las dos investigadoras y no por otros medios, con el fin de asegurar la fiabilidad y validez de los instrumentos en la recogida de la información.

El período de recogida de datos se efectuó empleando técnicas de persuasión y comunicación, para sincronizar de una manera más efectiva con los agentes objeto de estudio y su sensibilización por la investigación a desarrollar, como en el esfuerzo físico y los costes derivados del trabajo realizado.

Efectuado el trabajo de campo y recogidos todos los protocolos, en total 137, se procedió a la codificación e informatización de los datos en una matriz de datos Excel. Esta matriz se transformó en una matriz de SPSS versión 13.0 disponible en la ULE para la realización de los análisis estadísticos, efectuados por el I.P. (J.N. García). Luego de proceder con los análisis a través del SPSS, se procedió a la elaboración de tablas, gráficas y otros datos utilizables para esta memoria en la presentación de los resultados y poder aportar las evidencias empíricas de este estudio.

Seguidamente, se realizó la escritura de la memoria. Cabe destacar que la parte teórica de este proceso se inició antes de todo el desarrollo de la parte empírica y se continuó hasta el final de ésta. Concluida la escritura de la memoria, en la parte teórica, se hizo la escritura de la parte empírica, incluyendo la interpretación de los datos, la identificación y extracción de conclusiones, la explicitación de las limitaciones y las perspectivas futuras del estudio.

\section{RESULTADOS}

Los análisis y resultados que a continuación se exponen se centran en cuatro puntos específicos, (i) el análisis de las propiedades psicométricas del PRAES, que incluye la fiabilidad por consistencia interna y la validez, tanto del contenido como del constructo; (ii) el análisis de los datos descriptivos que destacan las medias de las medidas y de las variables; (iii) el análisis de regresión lineal (modelos de regresión lineal); y, (iv) los análisis multivariados de la varianza (modelos lineales generales). 


\section{Propiedades psicométricas del instrumento}

Se calculó la fiabilidad por consistencia interna de las escalas. Cuando se calcula la fiabilidad por consistencia interna del conjunto de los cuatro instrumentos, el $\alpha$ de Cronbach es de ,793, lo cual representa una fiabilidad aceptable. Cuando se calcula esta fiabilidad para cada uno de los cuatro instrumentos, los resultados son variables, siendo adecuados en general excepto para el PRAESPE con un $\alpha$ de Cronbach de ,581, lo cual es algo bajo; siendo para el resto de las escalas un $\alpha$ de Cronbach de: PRAESPO $=, 861$; para el PRAESPA $=, 788 ; \mathrm{y}$, para el PRAESAE $=, 843$.

Para estudiar la validez del instrumento PRAES, se llevaron a cabo análisis de validez de contenido o aparente y de constructo.

Validez de contenido del PRAES. De la revisión de estudios empíricos y teóricos internacionales, publicados en los últimos años, podemos afirmar de forma razonable que el PRAES presenta una validez de contenido o aparente adecuada. De esta revisión, podemos sintetizar los hallazgos y las evidencias empíricas, así como las conceptualizaciones teóricas, en cuatro grandes componentes que se refieren al papel de la práctica de los profesores en la enseñanza de la escritura.

El primer componente, el PRAESPO, hace referencia a la opinión que tienen los profesores en relación a los múltiples componentes de la enseñanza de la composición escrita como, por ejemplo, la motivación, la planificación y revisión, la familia, las prácticas y la formación del profesorado. Este tipo de instrumento, basado en la obtención de la opinión de los profesores, ha sido utilizado previamente, en parte, por Graham et al. (2001).

El segundo componente, PRAESPE, hace referencia al enfoque teórico que siguen los profesores en esta enseñanza, el cual se puede dicotomizar en dos grandes polos a lo largo de un continuo. En un polo, se sitúa basado en la enseñanza formal y, en el otro polo, se sitúa basado en el aprendizaje natural. Entendiendo que, a lo largo del continuo, los diferentes profesores se sitúan o presentan algunos elementos de esos enfoques. Este tipo de componente ha sido estudiado, previamente, en varias investigaciones de Graham et al. (2001).

Por otra parte, el componente relativo a las conductas concretas que realiza el profesor en el aula en la enseñanza de la composición escrita, PRAESPA, tiene que ver con elementos que incluyen el despliegue de habilidades, el uso de procedimientos específicos, el desempeño de actividades varias, el uso de materiales adecuados o la utilización de géneros textuales diversos. Algunos de estos aspectos fueron medidos y estudiados por Graham et al. (2001).

Por último, el componente de la auto-percepción de eficacia que tiene el profesor respecto a su desempeño como instructor-docente de la composición escrita, PRAESAE. Este componente hace referencia a la autoeficacia percibida por el 
profesor, se ajusta al constructo de Bandura (2005) y sigue sus directrices en la construcción de esa prueba.

Validez de constructo del PRAES. Se obtuvo el cálculo de la validez de constructo mediante el análisis factorial de componentes principales con rotación varimax normalizada.

El análisis permitió la extracción de cinco factores o componentes principales que, sólo en parte, se ajustan a la estructura de la prueba, siguiendo la validez de contenido elaborada en base a la revisión de estudios empíricos y teóricos internacionales. En general, los factores que se extraen del análisis no coinciden exactamente con los cuatro componentes del PRAES; aunque podemos decir que se aproximan, en gran medida, indicando, al menos parcialmente, una validez de constructo aceptable.

En primer lugar, se obtiene un factor que se ha denominado general, porque incluye saturaciones de los pesos factoriales de los totales de varias de las escalas del PRAES. Este factor general explica el 33,20\% de la varianza predicha total y está saturado por los pesos factoriales correspondientes al PRAESPO, subtotal de motivación $(-, 738)$ y subtotal de familia $(-, 882)$; más el PRAESPA con los subtotales de textos $(, 765)$ y de actividades $(, 813)$; además de los totales de autoeficacia personal $(, 569)$ y autoeficacia general $(, 479)$, aunque, en este último caso, como se ve, son de menor magnitud.

En segundo lugar, se obtiene un factor que se puede denominar enfoque teórico y de autoeficacia porque incluye saturaciones de los pesos factoriales de los totales de varias de las escalas del PRAESAE. Este factor explica el 22,49\% de la varianza total y está saturado por los pesos factoriales correspondientes al PRAESPE, subtotal de enseñanza formal $(, 929)$ y subtotal de aprendizaje natural $(-, 582)$; más el PRAES-PA con los subtotales de habilidades $(, 905)$ y de materiales $(, 446)$; además de los totales de autoeficacia personal $(, 657)$ y autoeficacia general $(, 580)$. Estos pesos factoriales más bajos contribuyen en menor medida a la configuración de este factor.

En tercer lugar, se obtuvo un factor que se ha denominado opinión puesto que incluye saturaciones de los pesos factoriales de los totales de varias de las escalas del PRAESPO. Este factor explica el 16,22\% de la varianza total y está saturado por los pesos factoriales correspondientes al PRAESPO, subtotal de práctica $(, 875)$ y subtotal de formación del profesorado $(, 896)$; más el PRAESPE con el subtotal de aprendizaje natural (,640).

En cuarto lugar, se obtiene otro factor al que se puede denominar, actividades, debido a que incluye saturaciones de los pesos factoriales de los totales de varias de las escalas del PRAESPA. Este factor explica el 8,80\% de la varianza total y está saturado por los pesos factoriales que corresponden al PRAESPA, subtotal de procedimiento (,978) y subtotal de materiales (,649); más el PRAESPO con el subtotal 
de motivación $(, 442)$. Este último con menor peso y contribuye en menor escala a la configuración de este factor.

En quinto lugar, se obtuvo el factor que se denominó planificación-revisión debido a que incluye saturaciones de los pesos factoriales de los totales en una de las escalas del PRAESPO. Este factor explica el 7,23\% de la varianza total y está saturado por los pesos factoriales del PRAESPO, subtotal de planificación y de revisión (,939); más el PRAESAE con el subtotal de autoeficacia general $(-, 408)$.

\section{Datos descriptivos}

En este apartado, se destacan las medias de las medidas y variables, tal y como se adjunta en la tabla 3, con el fin de hacerse una idea general de los resultados descriptivos. En esta tabla, figuran los datos relativos al número de participantes, las puntuaciones mínimas y máximas de cada variable, así como las medias y desviaciones típicas respectivas, además de las puntuaciones máximas para cada una de las variables.

Figura 1. Medias de las diversas variables analizadas en el estudio
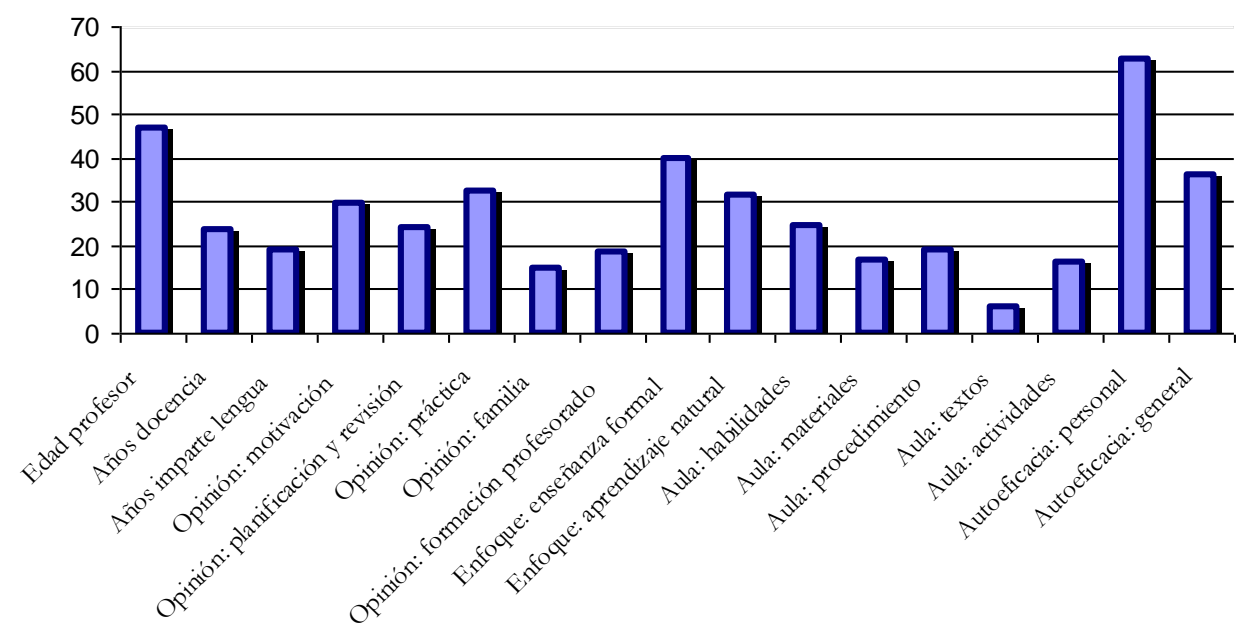

Como puede observarse en la tabla 3 y en la figura 1, en general, los datos indican una magnitud positiva en todas y cada una de las variables relativas a los instrumentos. Por ejemplo, en el PRAESPO, subcomponente de motivación, la media que se obtiene es de 29,65, que supera la mitad posible en esa variable, con una puntuación máxima de 35. Por otro lado, la media de docencia de los profesores es de 23,43 años, lo que indica una gran experiencia docente, siendo ésta en el área de Lengua de 18,84 años, lo cual hace suponer que son muy conocedores de los aspectos 
instruccionales relacionados, probablemente, con la enseñanza de la composición escrita.

Tabla 3. Estadísticos descriptivos de las variables generales e instrumentos

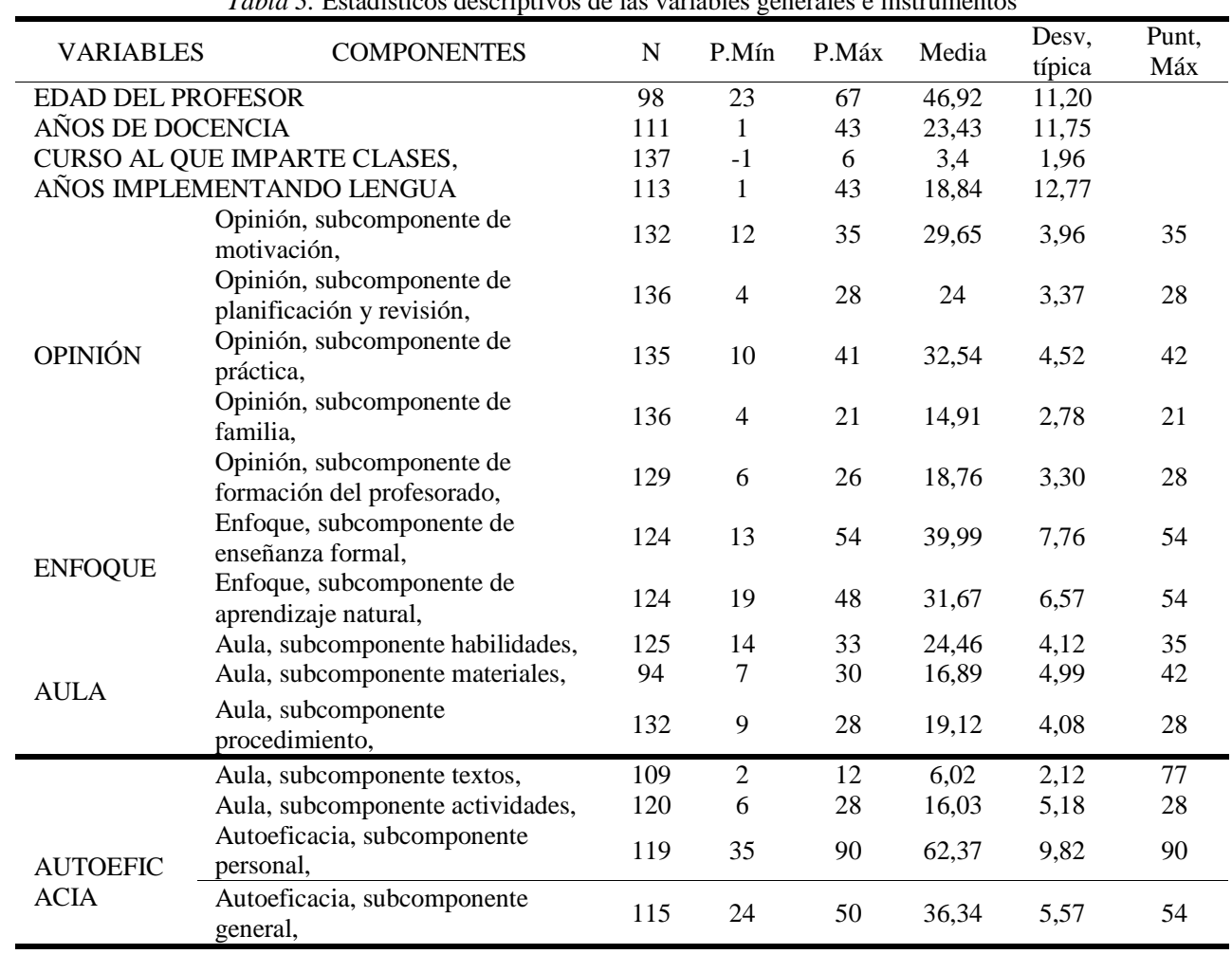

Como se ve, la edad media de los profesores es de 46,92 años, con un máximo de 67 y un mínimo de 23 años, indicando madurez y años de estabilidad en la docencia.

\section{Análisis de regresión lineal múltiple}

Se intenta predecir la variable dependiente (variable predicha) a partir del resto de las variables predictoras. Se toman como variables predictoras los totales de las cuatro escalas y se va tomando como variables predichas cada una de ellas, incluyendo otras variables de identificación como, por ejemplo, el curso en que imparte clases el profesor.

Cuando se toma como variable dependiente el curso en que imparte clases el profesor o variable predicha, a partir del conjunto de las medidas del PRAES y sus 
subescalas, en el análisis de regresión múltiple jerárquico, paso a paso, se obtiene un único modelo con un coeficiente de regresión significativo estadísticamente $\left(\mathrm{R}^{2}\right.$ corregida $\left.=, 313\right)$. Encontrando una única variable incluida en el modelo y es la del PRAESPA y el subcomponente procedimiento $[\beta=, 613, \mathrm{t}=-2.452, \mathrm{p}=, 034]$, quedando el resto de las variables excluidas del modelo al no alcanzar la significación estadística.

Por otra parte, cuando se intenta predecir el enfoque teórico formal del profesor, se obtiene un $\mathrm{R}^{2}$ corregida de, 616 , lo cual es muy alto aunque sólo se genera un modelo o ecuación de regresión con la variable predictora PRAESPA, subcomponente de habilidades $[\beta=, 807, \mathrm{t}=4.315, \mathrm{p}=, 002]$, no dando ninguna otra variable significativa, por lo que quedan excluidas del modelo.

Igualmente, la predicción del enfoque teórico natural del profesor es posible realizarlo con dos ecuaciones de regresión. El primer modelo alcanza un $\mathrm{R}^{2}$ corregida de, 331 , siendo significativa la variable de autoeficacia personal $[\beta=-, 626, t=-2.540$, $\mathrm{p}=$,029]; $\mathrm{y}$, en el segundo modelo, se alcanza un $\mathrm{R}^{2}$ corregida de ,556, siendo significativas, además de la autoeficacia personal del primer modelo, el PRAESPA y el subcomponente textos $[\beta=, 774, \mathrm{t}=2.465, \mathrm{p}=, 036]$, quedando excluidas el resto de las variables en el análisis de regresión múltiple paso a paso.

En síntesis, el análisis de regresión proporciona datos estadísticamente significativos en la predicción de todas y cada una de las variables del PRAES a partir del resto de las variables, lo que representa una medida de gran interés en la validez predictiva. Además, indica el gran potencial predictivo del PRAES ya que permite conocer unas variables que no siempre son de la misma naturaleza del resto. Por ejemplo, se incluye en el PRAES un subcomponente de opinión, uno de autoeficacia, otro que destaca el tipo de actividades o conductas que realizan los profesores en el aula y otro del enfoque teórico que siguen los profesores en la enseñanza de la escritura. Esta naturaleza diferente, en el que los coeficientes de regresión son tan altos, es un buen indicador que apoya la bondad del PRAES.

\section{Análisis multivariado de la varianza (modelos lineales generales)}

Es importante destacar que, el análisis para diferenciar el papel o la influencia que tienen las diferentes variables de agrupamiento (género de los profesores, años de docencia, centros, y, curso que imparte el profesor), no alcanza la significación estadística en los contrastes multivariados. Es decir, cada una de las variables del PRAES utilizadas como factores intersujetos, no alcanzan por sí mismas la significación estadística en los contrastes multivariados, excepto para el caso del enfoque teórico natural del profesor. Esta variable, al ser agrupada en tres conglomerados, con un contraste multivariado de la varianza, resulta estadísticamente significativa además de presentar un tamaño del efecto grande $[\lambda=, 001$; 
$\left.\mathrm{F}(2,18)=46.465, \mathrm{p}=, 021, \mathrm{y}^{2}=, 998\right] ;$ al igual que la variable autoeficacia personal cuando se agrupa en tres conglomerados y un tamaño del efecto grande $[\lambda=, 001$, $\left.\mathrm{F}(1,10)=1169.051, \mathrm{p}=, 023, \mathrm{y}^{2}=1\right]$.

Las pruebas de los efectos intersujetos de la autoeficacia personal y del enfoque teórico natural indican algunas variables significativas (ver tabla 4).

Tabla 4. Resultados del análisis multivariado de la varianza de las variables estadísticamente significativas del PRAES en tres conglomerados

\begin{tabular}{llcccc}
\hline VARIABLES SIGNIFICATIVAS & $\lambda$ & $\mathrm{F}(2,18)$ & $\mathrm{p}$ & $\mathrm{j}^{2}$ \\
\hline & & 0,001 & 46,465 & 0,021 & 0,998 \\
& Enfoque teórico formal & & & 0,053 & 0,480 \\
Enfoque teórico natural & PRAESPA: Subcomponente Habilidades & & 0,024 & 0,564 \\
& Enfoque teórico natural & & 0,001 & 0,938 \\
& Autoeficacia personal & $\Lambda$ & $\mathrm{F}(1,10)$ & $\mathrm{p}$ & $\mathrm{\eta}^{2}$ \\
\hline & & 0,001 & 1169,051 & 0,023 & 1 \\
& & & 0,038 & 0,365 \\
& PRAESPO: Subcomponente Familia & & 0,092 & 0,258 \\
Autoeficacia Personal & PRAESPA: Subcomponente Materiales & & & 0,001 & 0,779 \\
\hline & PRAESPA: Subcomponente Textos & & & 0,002 & 0,626 \\
\hline
\end{tabular}

En lo concerniente al enfoque teórico natural, las pruebas de los efectos intersujetos indican significación estadística para las variables enfoque teórico formal $\left(\mathrm{p}=, 053, \mathrm{p}^{2}=, 480\right)$, para el PRAESPA y el subcomponente habilidades $(\mathrm{p}=, 024$, $\left.\mathrm{y}^{2}=, 564\right)$, el mismo enfoque teórico natural sin agrupamientos, como era de esperar $\left(\mathrm{p}=, 001, \mathrm{y}^{2}=, 938\right)$, así como la autoeficacia personal $\left(\mathrm{p}=, 024, \mathrm{y}^{2}=, 565\right)$, tal y como se muestra en la figura 2 .

Figura 2. Análisis multivaridado: enfoque teórico natural (contraste multivariado)

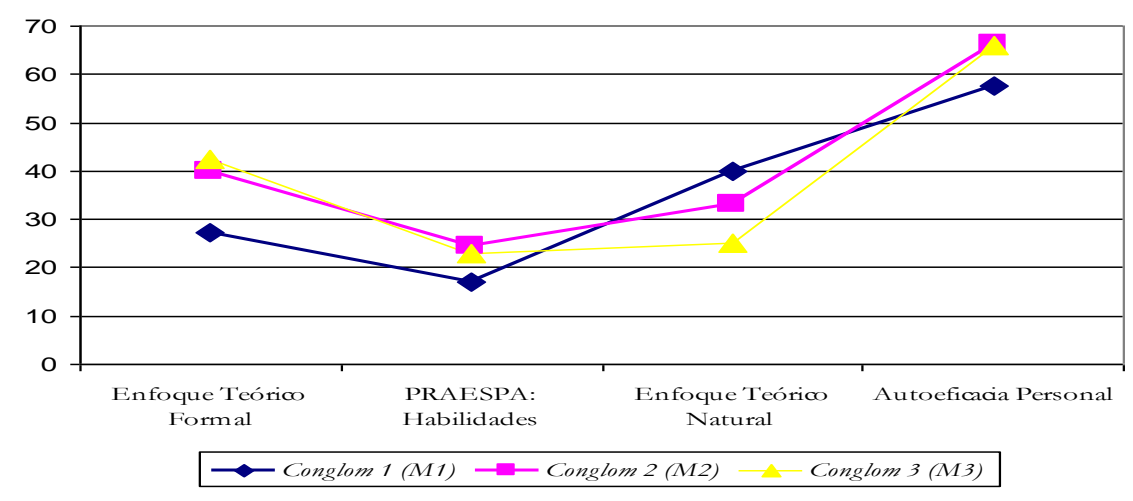

En las pruebas de los contrastes post hoc, sin embargo, se aprecian diferencias significativas entre los conglomerados de esta variable, en general. 
En lo que hace referencia a la autoeficacia personal, las pruebas de los efectos intersujetos indican significación estadística para las variables PRAESPO, subcomponente familia ( $\left.\mathrm{p}=, 038, \mathrm{n}^{2}=, 365\right)$; PRAESPA, subcomponente materiales $\left(\mathrm{p}=, 092, \mathrm{y}^{2}=, 258\right)$, si bien, en este caso, próximo a la significación estadística; también para el subcomponente textos $\left(\mathrm{p}=, 001, \mathrm{y}^{2}=, 779\right)$; $\mathrm{y}$, para la autoeficacia personal $\left(\mathrm{p}=, 002, \mathrm{y}^{2}=, 626\right)$. Ver figura 3 .

Figura 3. Análisis multivariado: autoeficacia personal (contraste multivariado)

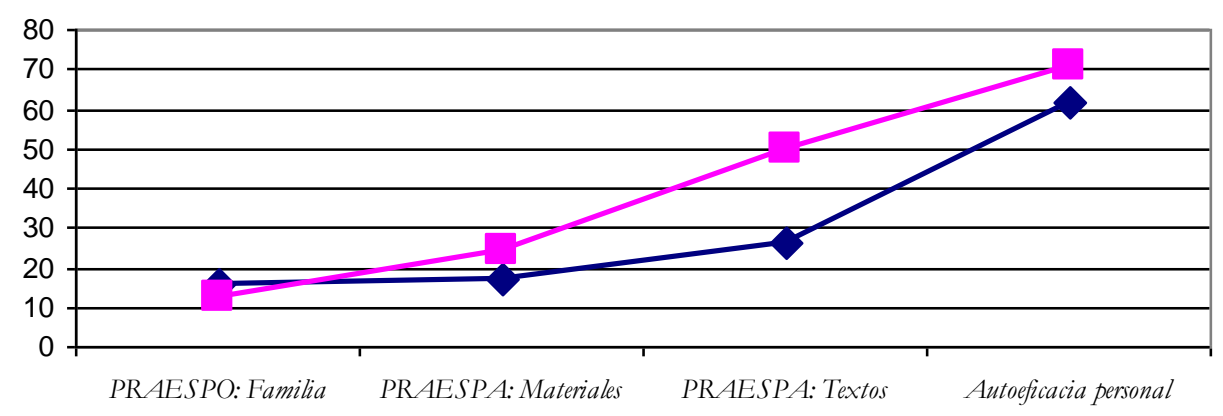

$\sim$ Conglom 1 (M1) Conglom 2 (M2)

\section{DISCUSION}

El objetivo del presente estudio fue considerar el tipo de práctica que llevaban a cabo los profesores del área de Lengua de la provincia de León en la enseñanza de la escritura, específicamente, en lo que concierne a los componentes que influyen en la práctica y las orientaciones teóricas que tienen los profesores, en torno al proceso de enseñanza-aprendizaje de la composición escrita, además de lo que hace a sus creencias de capacidad o autoeficacia, así como a las opiniones sobre los factores que inciden en la práctica del profesor en la enseñanza de la escritura.

Tomando en cuenta este objetivo, se esperaba que, producto de la revisión teórica de los estudios empíricos, el tipo de prácticas que los profesores desarrollan en clase eran determinadas y obedecían a factores como: la concepción teórica que tienen de cómo aprenden los alumnos, la forma de pensar sobre los efectos de estas instrucciones, la corrección de la escritura y los métodos de enseñanza (formal y natural), etc.

De acuerdo a los resultados obtenidos y, de manera general, se puede afirmar que el objetivo ha sido conseguido, puesto que se ha abordado el estudio del papel de la práctica, se han implementado instrumentos adecuados para su estudio, así como el haber utilizado una muestra representativa y relativa a un espectro de niveles 
educativos interesantes. Pero la hipótesis, sólo se ha confirmado parcialmente. Por una parte, podemos afirmar que el instrumento PRAES, implementado por nosotros, reúne los requisitos adecuados en cuanto a fiabilidad y validez, permitiendo la extracción de datos relevantes para el estudio del papel de la práctica. Pero no se cumple lo relativo al papel diferencial de los datos obtenidos de los maestros, en función del nivel educativo de los alumnos, puesto que no se obtienen diferencias estadísticamente significativas que indiquen una flexibilización y matización de las opiniones de los profesores, el enfoque teórico que asumen, lo que dicen que hacen o las creencias en su capacidad para enseñar escritura, en función de las características de los alumnos (en este caso, del progreso del alumno reflejado por el curso en que está).

El hecho de que los profesores indiquen que hacen lo mismo, opinan lo mismo, con el mismo esquema teórico de enseñanza de la escritura, y con los mismos sentimientos de capacidad, sin distinguir el nivel de los alumnos, puede tener varias explicaciones. Por una parte, puede que sea una constatación llana y simplemente de lo que efectivamente es esperable, que contradice nuestra hipótesis, y es que los profesores aplican los mismos esquemas sin considerar las características de los alumnos, porque su formación no sea específica o porque apenas se practica la instrucción en composición escrita, excepto en los niveles iniciales, con predominio del énfasis en los aspectos más mecánicos. Pero es posible que, al tratarse de medidas tan generales y no medidas directas de las actuaciones de los profesores, lo que reflejen sea deseabilidad de los profesores, incluyendo su propia ideología, sus creencias y teorías, sus opiniones, todas ellas muy genéricas, que al abordar en realidad la enseñanza de la escritura a alumnos de diferentes niveles sí que podrían encontrarse grandes diferencias puesto que no es posible enseñar de la misma manera a los alumnos de diferentes niveles educativos. En este sentido, el PRAES mediría más deseabilidad general o creencias generales que el papel de la práctica concreta. El instrumento se aplica al margen de la actividad directa de los profesores, es por ello que refleja componentes diríamos de "rasgo", más que de "situación". ¿Qué hubiera ocurrido si se hubiera observado a los profesores y maestros directamente en las clases de instrucción de la escritura con sus alumnos de diferentes niveles educativos? Es previsible que mostraran estrategias diferenciales, pero esta posibilidad ha de ser explorada con otro estudio.

En cuanto a la muestra, además de representativa, relevante y de amplio espectro (al ser comparada con las muestras de otros estudios empíricos revisados), permite describir cómo es el tipo de práctica que llevan a cabo estos profesores, y obtener datos valiosos, aunque, como acabamos de indicar, en parte limitados.

En cuanto al instrumento aplicado, dada la revisión de estudios empíricos y teóricos publicados en los últimos años, se puede afirmar de forma razonable que el PRAES presenta una adecuada validez y aceptable fiabilidad. De estas revisiones, de acuerdo a los hallazgos y las evidencias empíricas, además de la inexistencia de datos 
en el idioma español. No obstante, el cuestionario es el instrumento más utilizado en las investigaciones realizadas en esta dirección. Además, se puede asegurar que la construcción del PRAES ha recogido de forma selectiva y representativa los elementos a los que se refieren los componentes de Opinión, o Enfoque, el relativo a las conductas concretas que realiza el profesor en el Aula en la enseñanza de la composición escrita y al componente de Autoeficacia, anteriormente citados. Es preciso resaltar que no hay ningún estudio publicado que se conozca que utilice de forma conjunta la investigación de los cuatro componentes. Ello justifica y da relevancia a la realización de la presente investigación.

En cuanto al análisis estadístico y como aportación de la presente investigación, se obtuvieron cinco factores o componentes principales que, aunque sólo en parte se ajustan a la estructura de la prueba, tienen cierta coincidencia con los cuatro componentes del PRAES. Los nombres asignados a estos factores, por su relevancia con respecto a los cuatro componentes estudiados, fueron los siguientes.

En primer lugar, el factor general, el cual interrelaciona la autoeficacia, tanto personal como general del profesor, con la motivación, la familia y las actividades propias del aula en la enseñanza de la composición escrita. En segundo lugar, el factor de enfoque teórico y autoeficacia, puesto que interrelaciona el enfoque teórico formal y natural del profesor con las habilidades que desarrolla y los materiales que utiliza en la enseñanza de la escritura, determinando así su autoeficacia personal y general. Además, el tercer factor, denominado de opinión, que interrelaciona el enfoque teórico natural del profesor con su formación y la práctica que desarrolla en la enseñanza de la composición escrita. Igualmente, el cuarto factor o componente factorial de actividades, que interrelaciona la motivación con los procedimientos y los materiales utilizados en la enseñanza de la composición escrita. Y, por último, el factor de planificación y revisión, que relaciona la autoeficacia general con la planificación y revisión seguida por el profesor en la enseñanza de la escritura.

En síntesis, se puede afirmar que estos factores permiten inferir cómo es la práctica que desarrollan los profesores en el área de lengua. No encontrándose, como dato notable, diferencias significativas en relación a la concepción teórica y estrategias que aplican en la enseñanza de la escritura en los diferentes ciclos de la educación infantil y primaria.

Además, las bases curriculares explícitas en los Reales Decretos no parecen afectar esta práctica, puesto que, según los resultados obtenidos, todos parecen actuar de manera homogénea en cuanto a concepciones teóricas que les orientan. Lo cual indica, también, que no existe diferencia alguna en el tratamiento de la enseñanza y el aprendizaje de la escritura en alumnos con y sin dificultades de aprendizaje y/o bajo nivel de rendimiento. 
Al establecer comparaciones con otros estudios empíricos que abordan el mismo eje de interés, se encuentran aportaciones relevantes de esta investigación. En primer lugar, se trata del primer estudio de estas características en español y en nuestro entorno cultural, que conozcamos. En segundo lugar, por primera vez se abordan los cuatro componentes en un único instrumento (Opinión, Enfoque, conductas en el Aula y Autoeficacia), puesto que, en otras investigaciones de este mismo ámbito, sólo han estudiado uno ó dos aspectos. Por otra parte, la muestra o población objeto de estudio es bastante representativa, tanto por su amplitud como por los niveles educativos abarcados en esta investigación, ya que, según se observa, en los estudios empíricos revisados las muestras o son escasas o se centran en pocos niveles educativos.

En este mismo orden de ideas, cabe destacar las limitaciones propias del estudio. Señalando algunas que, de antemano y producto de la revisión teórica, se sabía se podían encontrar. La inexistencia de datos sobre el rendimiento de los alumnos de estos profesores para establecer relaciones significativas entre lo que el profesor enseña y qué/cómo aprenden los niños, es una de estas limitaciones, cuestión que persigue solventarse con el segundo estudio empírico.

Además, la investigación se hizo en base a cuestionarios con las limitaciones que tiene este tipo de instrumentos. Es bien sabido que, desde el punto de vista psicológico, se puede producir el efecto aquiescencia, es decir, la tendencia a puntuar positivamente a todo lo que se les pregunta. Aunque se controló esta variable introduciendo diferentes direcciones en los elementos del instrumento, probablemente haya que controlarlo con mayor eficacia.

Otra limitación, señalada también por otros autores, es la no observación directa de la actuación de estos profesores en el aula para comprobar efectivamente lo que dicen que hacen. La superación de esta limitación exigirá la implementación de estudios adicionales.

Así mismo, sería pertinente verificar si existen o no diferencias en los métodos de enseñanza de otras áreas. Esto sería de gran interés para detectar si se aplican las mismas estrategias, tanto con alumnos con y sin dificultades de aprendizaje, como en los diferentes niveles educativos.

\section{Agradecimientos}

Durante la realización de esta investigación se recibieron ayudas de la DGIMEC, proyecto de investigación competitivo SEJ2007-66898-EDUC (2007-2010), con fondos FEDER de la Unión Europea.

\section{REFERENCIAS}

Bandura, A. (2005). Guide for constructing self-efficacy scales (Revised). Available from Frank 
Pajares. Emory University.

Bain, J., Mills, C., Ballantyne, R. \& Packer, J. (2002). Developing Reflection on Practice Through Journal Writing: impacts of variations in the focus and level of feedback. Teachers and Teaching: theory and practice, 8(2), 171-196.

García, J.N. y Arias-Gundín, O. (2004). Intervención en estrategias de revisión del mensaje escrito. Psicothema, 16(2), 194-202.

García, J.N. \& Arias-Gundín, O. (2005a). Intervention in the writing revision process by jeans of social support in low achievement students. Poster presentado en la $29^{\text {th }}$ Annual IARLD Conference, Valencia.

García, J.N. \& Arias-Gundín, O. (2005b). Are the writing processes affected by the revision condition displayed (mechanic vs., substantive vs., mixed)? Symposium, 9th European Congress of Psychology, Granada.

García, J.N. y de Caso, A.M. (2002). Evaluación e intervención en la motivación hacia la escritura en niños con dificultades de aprendizaje. En J.N. García (Coord.), Aplicaciones de Intervención Psicopedagógica (pp. 131-148). Madrid: Pirámide.

García, J.N. y de Caso, A.M. (2002) ¿Es posible mejorar la productividad y coherencia de los textos escritos por alumnos con dificultades de aprendizaje y/o bajo rendimiento sin que cambie su disposición reflexiva hacía la escritura? Psicothema, 14(2), 457-461.

García, J.N. \& de Caso, A.M. (2004). Effects of a motivational intervention for improving the writing of children with learning disabilities. Learning Disabilities Quarterly, 27(3), 141159.

García, J.N. \& de Caso, A.M. (2005). Comparison of the effects on writing attitudes and writing self-efficacy of three different instructions in students with learning disabilities. International Journal of Educational Research. 43(4-5), 272-289.

García, J.N. \& de Caso, A.M. (2006a). Changes in writing self-efficacy and writing products and processes through specific training in self-efficacy beliefs of the learning disabled. Learning Disabilities: A Contemporary Journal, 4(2), 1-27.

García, J.N. \& de Caso, A.M. (2006b). The Role of Self-Efficacy Beliefs in Writing in Children with Learning Disabilities. In G.D. Sideridis \& D. Scanlon (Eds.), Proceedings of the $14^{\text {th }}$ World Congress on Learning Disabilities. A Multidisciplinary Approach to Learning Disabilities: Integrating Education, Motivation and Emotions (pp. 54-65). Boston: LDW.

García, J.N. y Fidalgo, R. (2003a). Diferencias en la conciencia de los procesos psicológicos de la escritura: Mecánicos frente a sustantivos y otros. Psicothema, 15(1), 41-48.

García, J.N. y Fidalgo, R. (2003b). Cambios metacognitivos de los procesos psicológicos de la escritura en alumnos de $3^{\circ}$ de EP a $3^{\circ}$ de ESO. Revista de Psicología General y Aplicada, 56(2), 239-253.

García, J.N. y Fidalgo, R. (2004). El papel del autoconocimiento de los procesos psicológicos de la escritura en la calidad de las composiciones escritas. Revista de Psicología General y Aplicada, 57(3), 181-298.

García, J.N. \& Fidalgo, R. (2006a). Effects of two types of self-regulatory instructions in students with learning disabilities in writing product, process and self-efficacy. Learning Disability Quarterly. 29(3), 181-211. 
García, J.N. \& Fidalgo, R. (2006b). A Comparative Study of Two Types of Self-Regulatory Writing Intervention Programs in Students with Learning Disabilities. In G.D. Sideridis $\&$ D. Scanlon (Eds.), Proceedings of the $14^{\text {th }}$ World Congress on Learning Disabilities. A Multidisciplinary Approach to Learning Disabilities: Integrating Education, Motivation and Emotions (pp. 77-88). Boston: LDW.

García, J.N. y González, L. (2006). Diferencias en la conciencia morfológica, la escritura y el lenguaje en función del desarrollo y el nivel educativo del niño. Psicothema, 18(2), 171179.

García, J.N. y Marbán, J. (2002). Instrucción estratégica en la composición escrita. Barcelona: Ariel.

García, J.N. y Marbán J. (2003). El proceso de composición escrita en alumnos con DA y/o BR: Estudio instruccional con énfasis en la planificación. Infancia y Aprendizaje, 25(4), 1-18.

García, J.N., Marbán, J. y de Caso, A.M. (2001). Evaluación colectiva de los procesos de planificación y factores psicológicos de la escritura (EPP y FPE). En J.N. García (Ed.), Dificultades de aprendizaje e intervención psicopedagógica (pp. 151- 155). Barcelona: Ariel.

García, J.N. \& Martínez, B. (2005). Influence the levels of working memory the writing composition processes in students with learning disabilities? En J.N. García (Symposium), The writing $\log$ in students with and without learning disabilities. Symposium invitado al $9^{\text {th }}$ European Congress of Psychology, Granada.

García, J.N., Pacheco. D.I., Díez, C., Robledo, P., Mtnez-Cocó, B., Rodríguez, C., González, L., de Caso, A.M., Fidalgo, R. \& Arias-Gundín, O. (2006). The role of the teacher's practice in writing related with writing product and process in students with and without LD. 15th Congress on Learning Disabilities Worldwide. Boston, Burlington: LDW.

García, J.N. y Rodríguez, C. (2007). Influencia del intervalo de registro y del organizador gráfico en el proceso y producto de la escritura y en otras variables psicológicas. Psicothema, 19(2), 198-205.

Graham, S., Harris, K., Fink, B. \& MacArthur, C. (2001). Teacher Efficacy in Writing: A Construct Validation With Primary Grade Teachers. Scientific Studies Of Reading, 5(2), 177-202.

Graham, S., Harris, K., McArthur, C. \& Fink, B. (2002). Primary grade teachers' theoretical orientations concerning writing instruction: Construct validation and a nationwide survey. Contemporary Educational Psychology, 27(2), 147-166.

Troia, G. (2006). Writing instruction for students with learning disabilities. In Ch. MacArthur, S. Graham \& J. Fitzgerald (Eds.), Handbook of Writing Research (pp. 324-336). New York, London: The Guilford Press.

Troia, G.A. \& Maddox, M.E. (2004). Writing instruction in middle schools: special and general education teachers share their views and voice their concerns. Exceptionality, 12(1), 1937.

Recibido: 28 de noviembre de 2008 Recepción Modificaciones: 4 de enero de 2009 Aceptado: 11 de enero de 2009 